\title{
Long-range correlation of thermal radiation
}

\author{
M. Patra and C. W. J. Beenakker \\ Instituut-Lorentz, Leiden University, P.O. Box 9506, 2300 RA Leiden, The Netherlands
}

(Received 4 September 1998)

\begin{abstract}
A general theory is presented for the spatial correlations in the intensity of the radiation emitted by a random medium in thermal equilibrium. We find that a nonzero correlation persists over large distances, compared to the transverse coherence length of the thermal radiation. This long-range correlation vanishes in the limit of an ideal black body. We analyze two types of systems (a disordered waveguide and an optical cavity with chaotic scattering), in which it should be observable. [S1050-2947(99)50501-5]
\end{abstract}

PACS number(s): 42.50.Ar, 42.25.Bs, 42.25.Kb, 42.50.Lc

The Hanbury-Brown-Twiss effect is the existence of spatial correlations in the intensity of thermal radiation by a distant source. It was originally proposed as an intensityinterferometric method to measure the angular opening of a star [1], far less susceptible to atmospheric distortion than amplitude-interferometric methods [2]. Two photodetectors at equal distance $r$ from a source (diameter $a$ ) will measure a correlated current if their separation $d$ is smaller than the transverse coherence length $d_{c} \simeq \lambda r / a$ of the radiation from the source at wavelength $\lambda$. The correlation function decays with increasing $d$ in an oscillatory way, with amplitude $\propto\left(d_{c} / d\right)^{3}[3]$.

The textbook results assume that the source of the thermal radiation is a blackbody, meaning that at each frequency any incident radiation is either fully absorbed or fully reflected. In a realistic system there will be a frequency range in which only partial absorption occurs. The purpose of this paper is to show that, in general, for thermal radiation the correlation function does not decay completely to zero, but to a nonzero $d$-independent background value. This long-range correlation is smaller than the short-range correlation by a factor $(\lambda / a)^{2}$, and becomes dominant for $d \gtrsim r(\lambda / a)^{1 / 3}$. It contains information on deviations of the thermal radiation from the blackbody limit.

The information contained in the long-range correlation is most easily described when the source is embedded in a waveguide (see Fig. 1). The waveguide has length $L$, crosssectional area $A \simeq a^{2}$, and supports $N=2 \pi A / \lambda^{2}$ propagating modes at frequency $\omega$, counting both polarizations. In the far-field, and close to normal incidence, each mode corresponds to a transverse coherence area $(r \lambda)^{2} / A \equiv d_{c}^{2}$. The source is in thermal equilibrium at temperature $T$. The radiation emitted through the left end of the waveguide is incident on a pair of photodetectors, one detecting the photocurrent $I_{k}(t)$ in mode $k$, and the other detecting $I_{l}(t)$. Each photocathode has an area equal to the coherence area or smaller. The photocount $n_{k}=\bar{n}_{k}+\delta n_{k}$ (number of photons counted in a time $t$ ) and the photocurrent $I_{k}=d n_{k} / d t=\bar{I}_{k}+\delta I_{k}$ fluctuate around their time-averaged values $\bar{n}_{k}$ and $\bar{I}_{k}=\bar{n}_{k} / t$. We seek the correlation function

$$
C_{k l}=\int_{-\infty}^{\infty} \overline{\delta I_{k}(t+\tau) \delta I_{l}(t)} d \tau=\lim _{t \rightarrow \infty} \frac{1}{t} \overline{\delta n_{k}(t) \delta n_{l}(t)} .
$$

The overbar indicates an average over many measurements on the same sample.

The advantage of embedding the source in a waveguide is that we can characterize it by a finite-dimensional scattering matrix $S(\omega)$, consisting of four blocks of dimension $N \times N$,

$$
S=\left(\begin{array}{cc}
r & t \\
t^{\prime} & r^{\prime}
\end{array}\right)
$$

A mode $l$, incident from the left, is reflected into mode $k$ with amplitude $r_{k l}$, and transmitted with amplitude $t_{k l}^{\prime}$. Similarly, $r_{k l}^{\prime}$ and $t_{k l}$ are the reflection and transmission amplitudes for a mode $l$, incident from the right. Reciprocity relates these amplitudes by $r_{k l}=r_{l k}, r_{k l}^{\prime}=r_{l k}^{\prime}$, and $t_{k l}=t_{l k}^{\prime}$.

It has been shown recently by one of the authors [4], using the method of "input-output relations" [5-7], how the photocount distribution can be expressed in terms of the scattering matrix. The expressions in Ref. [4] are for a single multimode photodetector. The corresponding formulas for two single-mode photodetectors are

$$
\begin{aligned}
C_{k l} & =\alpha_{k} \alpha_{l} \int_{0}^{\infty}\left|\left(Q Q^{\dagger}\right)_{k l}(\omega)\right|^{2}[f(\omega, T)]^{2} \frac{d \omega}{2 \pi}+\delta_{k l} \bar{I}_{k}, \\
\bar{I}_{k} & =\alpha_{k} \int_{0}^{\infty}\left(Q Q^{\dagger}\right)_{k k}(\omega) f(\omega, T) \frac{d \omega}{2 \pi},
\end{aligned}
$$

where $\alpha_{k}$ is the detector efficiency (the fraction of the photocurrent in mode $k$ that is detected), and $f$ is the BoseEinstein function

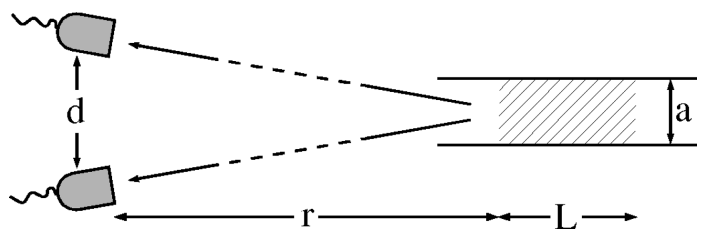

FIG. 1. Schematic diagram of a source (length $L$, diameter $a$ ) radiating into an $N$-mode waveguide that is open at both ends. The radiation leaving the waveguide at one end is detected by two photodetectors at a distance $r$ from the source, and separated by a distance $d$. The photocathodes have an area below the transverse coherence area $d_{c}^{2} \simeq r^{2} / N$. We find that the photocurrents are correlated even if the two detectors are separated by more than $d_{c}$. 


$$
f(\omega, T)=\left[\exp \left(\hbar \omega / k_{B} T\right)-1\right]^{-1}
$$

The $N \times N$ matrix $Q$ is related to the reflection and transmission matrices by

$$
Q Q^{\dagger}=1-r r^{\dagger}-t t^{\dagger}
$$

The integral over $\omega$ extends over a range $\Omega_{c}$ set by the absorption linewidth, centered at $\omega_{0}$. Typically, $\Omega_{c} \ll \omega_{0}$, so we can neglect the frequency dependence of $N$ and $f$. The matrix $Q(\omega)$ for a random medium fluctuates on a scale $\omega_{c}$ much smaller than $\Omega_{c}$. The integration over $\omega$ then averages out the fluctuations, so that we may replace the integrand by its ensemble average, indicated by \langle\rangle ,

$$
C_{k l}=\alpha_{k} \alpha_{l} f^{2} \int_{0}^{\infty}\left\langle\left|\left(Q Q^{\dagger}\right)_{k l}(\omega)\right|^{2}\right\rangle \frac{d \omega}{2 \pi}+\delta_{k l} \bar{I}_{k} .
$$

We evaluate the ensemble average using results from random-matrix theory [8]. For a medium with randomly placed scatterers, the "equivalent channel approximation", [9] has proven to be reliable. According to this approximation, all $N$ modes are statistically equivalent. As a consequence, for any $k \neq l$, one has

$$
\begin{aligned}
\left\langle\operatorname{tr}\left(Q Q^{\dagger}\right)^{2}\right\rangle & =N \sum_{j=1}^{N}\left\langle\left(Q Q^{\dagger}\right)_{k j}\left(Q Q^{\dagger}\right)_{j k}\right\rangle \\
& =N(N-1)\left\langle\left|\left(Q Q^{\dagger}\right)_{k l}\right|^{2}\right\rangle+N\left\langle\left(Q Q^{\dagger}\right)_{k k}^{2}\right\rangle .
\end{aligned}
$$

The average of $\left(Q Q^{\dagger}\right)_{k k}^{2}$ factorizes in the large- $N$ limit [8],

$$
\left\langle\left(Q Q^{\dagger}\right)_{k k}^{2}\right\rangle=\left\langle\left(Q Q^{\dagger}\right)_{k k}\right\rangle^{2}\left[1+\mathcal{O}\left(N^{-1}\right)\right]=N^{-2}\left\langle\operatorname{tr} Q Q^{\dagger}\right\rangle^{2} .
$$

The combination of Eqs. (7) and (8) gives us

$$
\left\langle\left|\left(Q Q^{\dagger}\right)_{k l}\right|^{2}\right\rangle=N^{-2}\left\langle\operatorname{tr}\left(Q Q^{\dagger}\right)^{2}\right\rangle-N^{-3}\left\langle\operatorname{tr} Q Q^{\dagger}\right\rangle^{2}+\mathcal{O}\left(N^{-2}\right) \text {. }
$$

The eigenvalues $\sigma_{1}, \sigma_{2}, \ldots, \sigma_{N}$ of the matrix $r r^{\dagger}+t t^{\dagger}$ are the "scattering strengths" of the random medium. We denote by $\overline{\sigma^{p}} \equiv N^{-1} \sum_{n} \sigma_{n}^{p}$ the $p$ th spectral moment of the scattering strengths. According to Eqs. (5), (6), and (9), the cross correlator $C_{k l}(k \neq l)$ then takes the form of a variance,

$$
C_{k l}=\frac{\alpha_{k} \alpha_{l} f^{2}}{N} \int_{0}^{\infty}\left(\left\langle\bar{\sigma}^{2}\right\rangle-\langle\bar{\sigma}\rangle^{2}\right) \frac{d \omega}{2 \pi} .
$$

This is our basic result for the long-range correlation announced in the introduction. The information contained in the cross correlator is the variance of the scattering strengths. The autocorrelator, in contrast, depends entirely on the first spectral moment,

$$
\begin{gathered}
C_{k k}=\alpha_{k}^{2} f^{2} \int_{0}^{\infty}\langle 1-\bar{\sigma}\rangle^{2} \frac{d \omega}{2 \pi}+\bar{I}_{k}, \\
\bar{I}_{k}=\alpha_{k} f \int_{0}^{\infty}\langle 1-\bar{\sigma}\rangle \frac{d \omega}{2 \pi},
\end{gathered}
$$

where we have used Eq. (8).

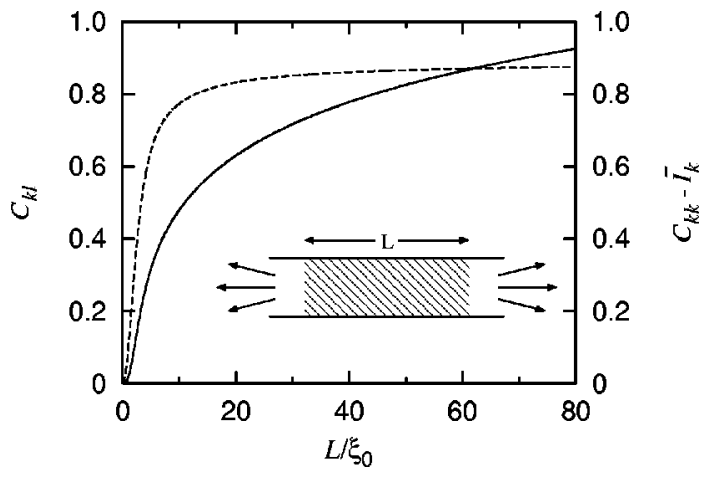

FIG. 2. Long-range correlation $C_{k l}$ (solid line), in units of $\Omega_{c} l f^{2} \alpha_{k} \alpha_{l} / N \xi_{0}$, and short-range correlation $C_{k k}-\bar{I}_{k}$ (dashed line), in units of $\Omega_{c}\left(l f \alpha_{k} / \xi_{0}\right)^{2}$, of the radiation emitted from a disordered waveguide (inset). A Lorentzian frequency dependence is assumed for the dielectric function, with width $\Omega_{c}$ and absorption length $\xi_{0}$ at the center of the absorption line. The mean-free-path $l$ is assumed to be $\ll \xi_{0}$. The short-range correlation saturates in the limit $L / \xi_{0}$ $\rightarrow \infty$, while the long-range correlation keeps increasing $\propto \ln L / \xi_{0}$.

The long-range correlation $C_{k l}$ of two photodetectors, separated by more than a coherence length, is an order $N$ smaller than the short-range correlation $C_{k k}-\bar{I}_{k}$ of two photodetectors separated by less than a coherence length. (The full value $C_{k k}$ is measured in a single-detector experiment.) The long-range correlation vanishes if all $N$ scattering strengths are the same, as they would be for an idealized "step-function model" of a blackbody $\left(\sigma_{n}=0\right.$ for $\left|\omega-\omega_{0}\right|<\Omega_{c}$, and $\sigma_{n}=1$ otherwise). A random, partially absorbing medium, in contrast, has a broad distribution of scattering strengths [8], hence a substantial long-range correlation of the photocurrent.

As a first example, we compute the correlation for a weakly absorbing, strongly disordered medium. The moments of $r r^{\dagger}$ and $t t^{\dagger}$, appearing in Eqs. (10) and (11), have been calculated by Brouwer [10] as a function of the number of modes $N$, the sample length $L$, the mean free path $l$, and the absorption length $\xi=\sqrt{D \tau_{a}}$ ( $\tau_{a}$ is the absorption time and $D=c l / 3$ is the diffusion constant). It is assumed that $1 / N \ll l / \xi \ll 1$, but the ratio $L / \xi \equiv s$ is arbitrary. The result is

$$
\begin{gathered}
\left\langle\bar{\sigma}^{2}\right\rangle-\langle\bar{\sigma}\rangle^{2}=\frac{2 l}{3 \xi}\left(\operatorname{coth}^{3} s-\frac{3}{\sinh s}+\frac{s}{\sinh ^{2} s}\right. \\
\left.+\frac{s \operatorname{coth} s-1}{\sinh ^{3} s}-\frac{s}{\sinh ^{4} s}\right), \\
\langle 1-\bar{\sigma}\rangle=\frac{4 l}{3 \xi} \tanh \frac{s}{2} .
\end{gathered}
$$

To compute the correlators (10) and (11) it remains to carry out the integrations over $\omega$. The frequency dependence is governed by the imaginary part of the dielectric function $\varepsilon^{\prime \prime}(\omega)$, for which we take the Lorentzian $\varepsilon^{\prime \prime}(\omega)=\varepsilon_{0}^{\prime \prime}[1$ $\left.+\left(\omega-\omega_{0}\right)^{2} / \Omega_{c}^{2}\right]^{-1}$. Since $\tau_{a}=1 / \omega_{0} \varepsilon^{\prime \prime}$, the corresponding $\omega$ dependence of $\xi$ and $s$ is $\xi / \xi_{0}=s_{0} / s=[1+(\omega$ $\left.\left.-\omega_{0}\right)^{2} / \Omega_{c}^{2}\right]^{1 / 2}$, with $\xi_{0}$ and $s_{0}$ the values of $\xi$ and $s$ at $\omega=\omega_{0}$. Results are plotted in Fig. 2. In the limit $L / \xi_{0} \rightarrow 0$ of 
a thin sample, we have

$$
\begin{gathered}
C_{k l}=\frac{1}{45} \Omega_{c}\left(l f^{2} \alpha_{k} \alpha_{l} / N \xi_{0}\right)\left(L / \xi_{0}\right)^{3}, \\
C_{k k}=\frac{4}{9 \pi} \Omega_{c}\left(l f \alpha_{k} / \xi_{0}\right)^{2}\left(L / \xi_{0}\right)^{2}+\bar{I}_{k}, \\
\bar{I}_{k}=\frac{1}{3} \Omega_{c}\left(l f \alpha_{k} / \xi_{0}\right)\left(L / \xi_{0}\right) .
\end{gathered}
$$

In the opposite limit $L / \xi_{0} \rightarrow \infty$ of a thick sample, the cross correlator $C_{k l}$ and the mean current $\bar{I}_{k}$ both diverge logarithmically $\propto \ln L / \xi_{0}$. The ratio $C_{k l} /\left(\bar{I}_{k} \bar{I}_{l}\right)^{1 / 2}$ tends to $(1 / 2 N) f \sqrt{\alpha_{k} \alpha_{l}}$ in the large- $L$ limit, and the short-range correlation $C_{k k}-\bar{I}_{k}$ tends to $\frac{8}{9} \Omega_{c}\left(l f \alpha_{k} / \xi_{0}\right)^{2}$, which remains larger than the long-range correlation because the limit $N$ $\rightarrow \infty$ has to be taken before $L \rightarrow \infty$.

Our second example is an optical cavity filled with an absorbing random medium [see Fig. 3(a), inset]. The radiation leaves the cavity through a waveguide supporting $N$ modes. The general formula (3) applies with $Q Q^{\dagger}=1-r r^{\dagger}$ (since there is no transmission). The scattering strengths $\sigma_{1}, \sigma_{2}, \ldots, \sigma_{N}$ in this case are eigenvalues of $r r^{\dagger}$. Their distribution is known in the large- $N$ limit [11] as a function of the dimensionless absorption rate $\gamma=2 \pi / N \tau_{a} \Delta \omega$, with $\Delta \omega$ the spacing of the cavity modes near frequency $\omega_{0}$. (The quantity $\gamma$ is the ratio of the mean dwell time in the cavity without absorption and the absorption time.) The moments $\langle\bar{\sigma}\rangle$ and $\left\langle\bar{\sigma}^{2}\right\rangle$ can then be computed by numerical integration. Results are shown in Fig. 3, again for a Lorentzian frequency dependence of $\varepsilon^{\prime \prime}(\omega)$. Unlike in the first example, we are now not restricted to weak absorption but can let the absorption rate $\gamma_{0}$ at the central frequency $\omega_{0}$ become arbitrarily large. For weak absorption, $\gamma_{0} \ll 1$, we have

$$
\begin{gathered}
C_{k l}=\frac{1}{4} \Omega_{c}\left(f^{2} \alpha_{k} \alpha_{l} / N\right) \gamma_{0}^{2}, \\
C_{k k}=\frac{1}{4} \Omega_{c}\left(f \alpha_{k} \gamma_{0}\right)^{2}+\bar{I}_{k}, \quad \bar{I}_{k}=\frac{1}{2} \Omega_{c} f \alpha_{k} \gamma_{0} .
\end{gathered}
$$

For strong absorption, $\gamma_{0} \gg 1$, all three quantities $C_{k l}, C_{k k}$ and $\bar{I}_{k}$ diverge $\propto \sqrt{\gamma_{0}}$ [see Fig. 3(a)]. The ratio $C_{k l} /\left(\bar{I}_{k} \bar{I}_{l}\right)^{1 / 2}$ tends to $0.062 f\left(\alpha_{k} \alpha_{l}\right)^{1 / 2} / N$, and the ratio $\left(C_{k k}-\bar{I}_{k}\right) / \bar{I}_{k}$ to $\frac{1}{2} f \alpha_{k}$ [see Fig. 3(b)]. The long-range correlation does not
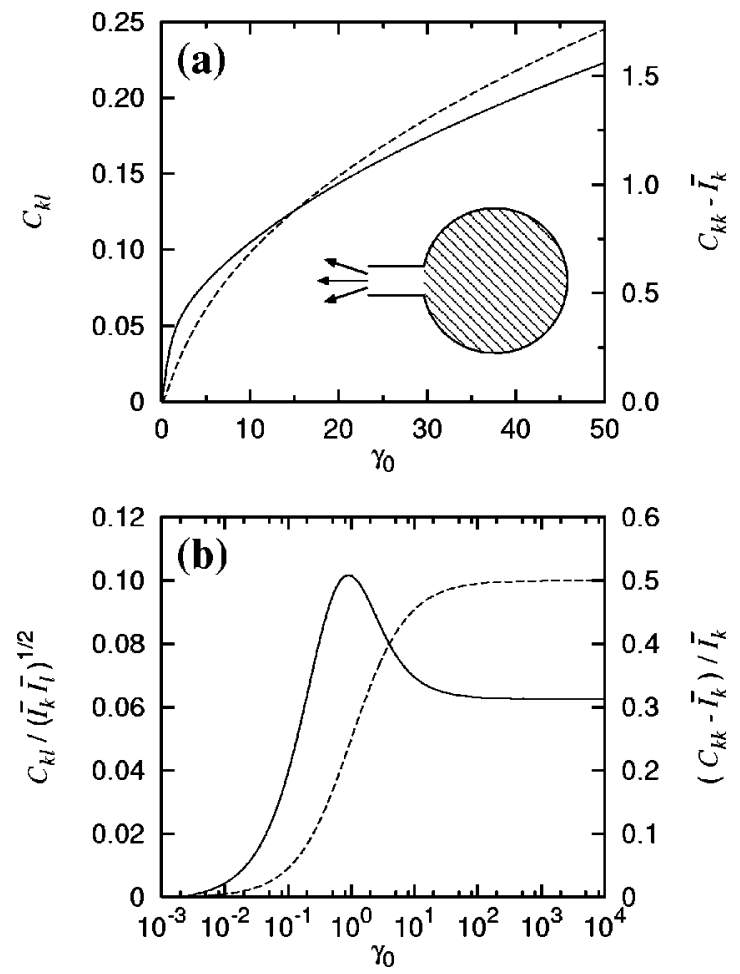

FIG. 3. Correlators of the radiation emitted from a disordered optical cavity (inset), as a function of the absorption rate $\gamma_{0}$, at the center of the absorption line with Lorentzian profile. (The absorption rate is normalized to the mean dwell time.) (a) Long-range correlation $C_{k l}$ (solid line), in units of $\Omega_{c} f^{2} \alpha_{k} \alpha_{l} / N$, and shortrange correlation $C_{k k}-\bar{I}_{k}$ (dashed line), in units of $\Omega_{c} f^{2} \alpha_{k}^{2}$. (b) Same correlators, but now normalized by the mean photocurrent. (The left axis is in units of $f \sqrt{\alpha_{k} \alpha_{l}} / N$; the right axis in units of $f \alpha_{k}$.) The long-range correlation persists in the limit $\gamma_{0} \rightarrow \infty$ because of partial absorption in the tails of the absorption line.

vanish as $\gamma_{0} \rightarrow \infty$, because there remains a tail of frequencies with moderate absorption and thus a wide distribution of scattering strengths, even if the system behaves like an ideal blackbody for frequencies near $\omega_{0}$.

In summary, we have shown that the thermal radiation emitted by random media contains long-range spatial correlations in the intensity. The long-range correlation has information on the spectral variation of the scattering strengths that is not accessible from the luminosity. We have analyzed two types of systems in detail, providing specific predictions that we hope will motivate an experimental search for the long-range correlation.

This work was supported by the Dutch Science Foundation NWO/FOM.
[1] R. Hanbury-Brown and R. Q. Twiss, Nature (London) 178, 1046 (1956).

[2] D. H. Boal, C.-K. Gelbke, and B. K. Jennings, Rev. Mod. Phys. 62, 553 (1990).

[3] L. Mandel and E. Wolf, Optical Coherence and Quantum Optics (Cambridge University Press, Cambridge, 1995).
[4] C. W. J. Beenakker, Phys. Rev. Lett. 81, 1829 (1998).

[5] J. R. Jeffers, N. Imoto, and R. Loudon, Phys. Rev. A 47, 3346 (1993).

[6] R. Matloob, R. Loudon, S. M. Barnett, and J. Jeffers, Phys. Rev. A 52, 4823 (1995)).

[7] T. Gruner and D.-G. Welsch, Phys. Rev. A 54, 1661 (1996). 
[8] C. W. J. Beenakker, Rev. Mod. Phys. 69, 731 (1997).

[9] P. A. Mello and S. Tomsovic, Phys. Rev. B 46, 15963 (1992).

[10] P. W. Brouwer, Phys. Rev. B 57, 10526 (1998). Equation (13c) contains a misprint: The second and third terms between brackets should have, respectively, minus and plus signs, instead of plus and minus.

[11] C. W. J. Beenakker, in Diffuse Waves in Complex Media, NATO Advanced Study Institute, Series E: Applied Sciences, edited by J. P. Fouque (Kluwer, Dordrecht, in press). 